\title{
Mehrfachbehandlung von Tätowierungen - bessere Aufhellung bei höherem Nebenwirkungsrisiko?
}

\author{
Multiple Exposure for Tattoo Removal - Better Clearance with Higher Risk \\ of Scarring?
}

Autoren

Institute
M. Drosner ${ }^{1,2}$, L. Trennheuser ${ }^{3}$

${ }^{1}$ Haut \& Laser Schwerin, Private Hautarztpraxis, Schwerin

${ }^{2}$ Dermatologische Klinik und Poliklinik der Technischen Universität, München

${ }^{3}$ Medizinische Fakultät der Universität Heidelberg
Bibliografie

DOI http://dx.doi.org/

$10.1055 / \mathrm{s}-0033-1344315$

Akt Dermatol 2013; 39: 283-287

(c) Georg Thieme Verlag KG

Stuttgart · New York

ISSN 0340-2541

Korrespondenzadresse Prof. Dr. med. Michael Drosner Haut \& Laser Schwerin Bischofstr. 11 19055 Schwerin info@hlzs.de

\section{Zusammenfassung \\ $\nabla$}

Einleitung: Die Entfernung von Tätowierungen mit gütegeschalteten Festkörperlasern ist effektiv und nebenwirkungsarm aber langwierig durch viele Wiederholungsbehandlungen. Um die Aufhellung zu beschleunigen, wurde kürzlich eine Methode der Vierfachbehandlung in einer Therapiesitzung beschrieben. Mit der vorliegenden Kasuistik sollen die ersten eigenen Erfahrungen mit dieser Mehrfachbehandlungsmethode beschrieben werden.

Patienten und Methode: Behandelt wurden $6 \mathrm{~Pa}-$ tienten mit schwarzen professionellen Tätowierungen. Alle erhielten eine Vierfachbehandlung in einem Probeareal und eine Einfachbehandlung der übrigen Tätowierung mit einem gütegeschalteten Nd:YAG-Laser $\left(1,064 \mathrm{~nm}\right.$, Spectra ${ }^{\mathrm{TM}}$, Lutronic Cooperation). Drei Patienten wurden erstmals behandelt. Der Gehalt an schwarzem Tätowierungspigment wurde vor und mindestens 8 Wochen nach der Behandlung fotodokumentiert und anhand einer 101-stufigen Grauwerttabelle durch 2 Personen gemessen.

\section{Einleitung}

Die fotoakustische Disruption mittels gütegeschalteter Festkörperlaser ist für die Entfernung von Tätowierungen seit den 90er-Jahren des vergangenen Jahrhunderts die Behandlungsmethode der ersten Wahl [1]. Bei der sehr komplexen Interaktion des sehr kurzen (Nanosekunden) und hochdosierten Lichtimpulses des Lasers wird das Pigment in kleinere Fragmente aufgespalten. Anschließend kommt es zum Abtransport der so zerkleinerten und phagozytierten Pigmentpartikel via Lymphsystem [2]. Die mittlere Anzahl notwendiger Wiederholungsbehandlungen für eine
Ergebnisse: Alle Patienten zeigten im Feld der Vierfachbehandlung eine bessere Aufhellung als in der übrigen Tätowierung ( $28 \%$ vs. $13,2 \%$ ). Ausgehend von einem Schwarzanteil von $80,5 \%$ vor der Behandlung, sind im Vierfachfeld 54\% und in der übrigen Tätowierung 68\% Schwarzanteil verblieben. Der Aufhellungseffekt konnte durch die Vierfachbehandlung im Mittel verdoppelt werden.

Bei 4 der 6 Patienten waren in den Probearealen der Mehrfachbehandlung Nebenwirkungen (Erythem, Strukturveränderung, Narben) aufgetreten, während die einfach behandelten Areale nebenwirkungsfrei behandelt wurden.

Schlussfolgerung: Der bessere Aufhellungseffekt durch eine mehrfache Behandlung von Tätowierungen in einer Sitzung konnte mit der vorliegenden Kasuistik bestätigt werden, auch wenn er nicht so deutlich ausfiel wie bei den Erstbeschreibern. Mit Nebenwirkungen bei 4 von 6 Patienten kann diese Variante der Tätowierungsbehandlung aus unserer Sicht noch nicht empfohlen werden, bevor weitere Untersuchungen die optimale Anzahl und Intervalle der Mehrfachbehandlungen geklärt haben.

zufriedenstellende Entfernung von Profitätowierungen variiert und wird mit 10-15 Wiederholungsbehandlungen angegeben [3,4], die gleichermaßen Geduld und Finanzen der Patienten strapazieren, zumal auch Fälle mit über 20 Sitzungen genannt werden. Durch eine Modifikation der Methode, bei der die Tätowierung in nur einer Sitzung viermal mit jeweils 20 Minuten Abstand wiederholt behandelt wird, soll sich die Tätowierungsentfernung auf meist nur eine Behandlungssitzung verkürzen lassen [5]. In der vorliegenden Kasuistik werden die ersten Erfahrungen der Anwendung dieser neuen Methode dargestellt und diskutiert. 
Tab. 1 Behandlungsparameter der jeweiligen Teilbehandlung der Tätowierungen mittels gütegeschaltetem Nd:YAG-Laser (Wellenlänge: 1,064 nm) im Rahmen der Mehrfachbehandlung.

\begin{tabular}{|c|c|c|c|c|c|c|c|c|c|}
\hline \multirow{2}{*}{$\begin{array}{l}\text { Pat. } \\
\text { Nr. }\end{array}$} & \multirow{2}{*}{$\begin{array}{l}\text { Behandlungs- } \\
\text { datum }\end{array}$} & \multicolumn{2}{|c|}{ 1.Teilbehandlung } & \multicolumn{2}{|c|}{ 2. Teilbehandlung } & \multicolumn{2}{|c|}{ 3. Teilbehandlung } & \multicolumn{2}{|c|}{ 4. Teilbehandlung } \\
\hline & & $\begin{array}{l}\text { Spotgröße } \\
(\mathrm{mm})\end{array}$ & $\begin{array}{l}\text { Energiedichte } \\
\left(\mathrm{J} / \mathrm{cm}^{2}\right)\end{array}$ & $\begin{array}{l}\text { Spotgröße } \\
(\mathrm{mm})\end{array}$ & $\begin{array}{l}\text { Energiedichte } \\
\left(\mathrm{J} / \mathrm{cm}^{2}\right)\end{array}$ & $\begin{array}{l}\text { Spotgröße } \\
\text { (mm) }\end{array}$ & $\begin{array}{l}\text { Energiedichte } \\
\left(\mathrm{J} / \mathrm{cm}^{2}\right)\end{array}$ & $\begin{array}{l}\text { Spotgröße } \\
(\mathrm{mm})\end{array}$ & $\begin{array}{l}\text { Energiedichte } \\
\left(\mathrm{J} / \mathrm{cm}^{2}\right)\end{array}$ \\
\hline 1 & 15.05 .12 & 3,0 & 7,0 & 3,0 & 8,0 & 3,0 & 9,0 & 3,0 & 9,0 \\
\hline 2 & 08.06 .12 & 4,0 & 4,5 & 4,0 & 6,0 & 4,0 & 5,0 & 4,0 & 5,0 \\
\hline 3 & 14.06 .12 & 5,0 & 1,1 & 5,0 & 1,7 & 5,0 & 3,2 & 5,0 & 4,0 \\
\hline 4 & 12.07 .12 & 4,0 & 1,2 & 4,0 & 1,8 & 4,0 & 4,5 & 4,0 & 6,5 \\
\hline 5 & 13.07 .12 & 3,0 & 6,0 & 3,0 & 7,0 & 3,0 & 7,5 & 3,0 & 8,0 \\
\hline 6 & 09.10 .12 & 8,0 & 1,2 & 6,0 & 2,2 & 6,0 & 3,6 & 5,0 & 4,5 \\
\hline \multicolumn{2}{|c|}{ Median } & 4,0 & 2,9 & 4,0 & 4,1 & 4,0 & 4,8 & 4,0 & 5,8 \\
\hline \multicolumn{2}{|l|}{ SD } & 1,9 & 2,7 & 1,2 & 2,9 & 1,2 & 2,3 & 0,9 & 2,0 \\
\hline
\end{tabular}

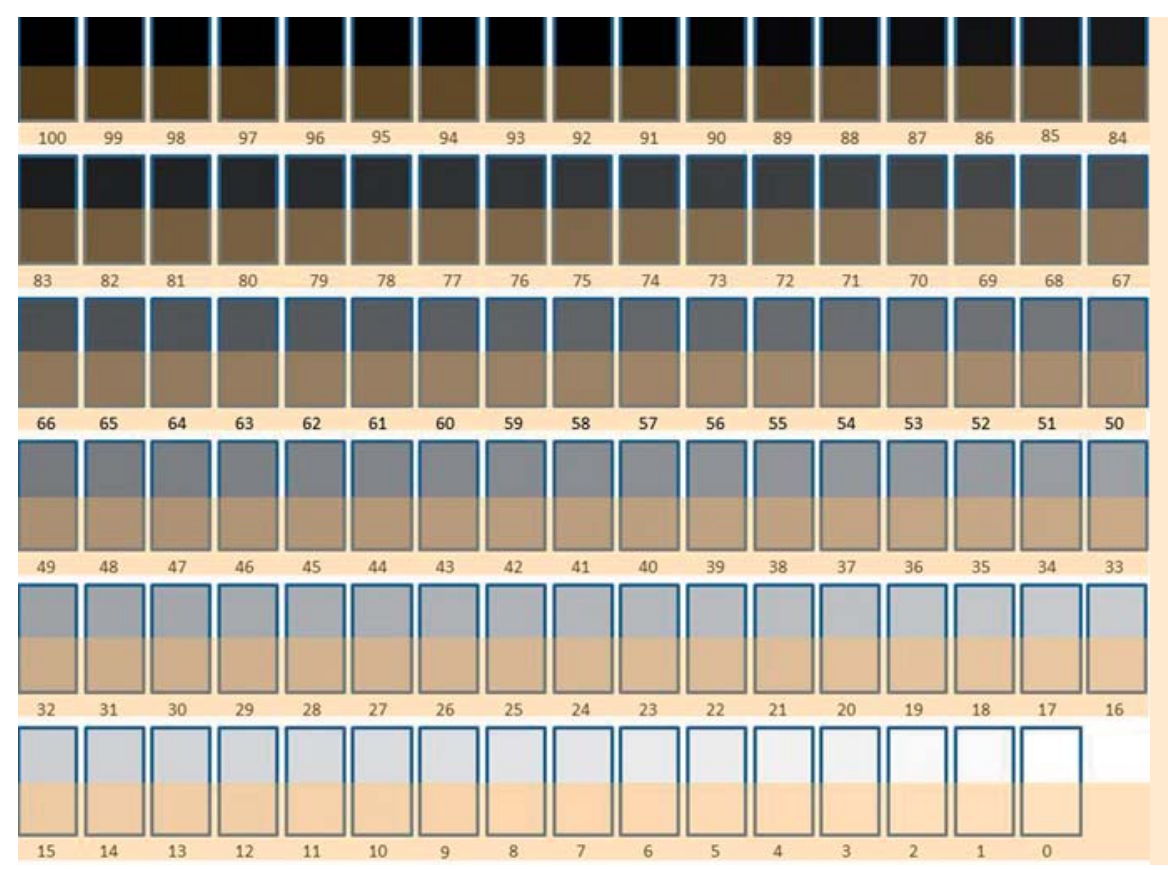

Abb. 1 Grauwerttabelle mit Schwarzanteilen in Prozent, jeweils zur Hälfte unterlegt mit einem hautfarbenen Braunton.

\section{Fallbericht}

$\nabla$

\section{Patienten und Methode}

Während 7 Monaten (Mai 2012 - Dezember 2012) wurden 6 Patienten (3 Frauen, 3 Männer, mittleres Alter: 34 Jahre) zur Entfernung von Profitätowierungen aus schwarzer Farbe behandelt und nachuntersucht. Nach Aufklärung über die Möglichkeit einer alternativen Behandlungsmethode, die möglicherweise schneller zur vollständigen Entfernung der Tätowierung führt, wurde mit Einverständnis der Patienten neben der Gesamtbehandlung der Tätowierung eine kleine Teilfläche zusätzlich drei weitere Male (=Vierfachbehandlung) mit mindestens 20-minütigem Abstand mit einem gütegeschalteten Nd:YAG-Laser (Spectra ${ }^{\mathrm{TM}}$, Lutronic Cooperation, Republik Korea) mit einer Wellenlänge von $1,064 \mathrm{~nm}$ behandelt. Parallel zur Behandlung wurde die Oberfläche mittels Kaltluftgebläse (Cryo mini ${ }^{\circledR}$, Zimmer MedizinSysteme $\mathrm{GmbH}$, Neu-Ulm) gekühlt. Unmittelbar vor der Behandlung und bei der Wiedervorstellung zur nächsten Behandlung wurde das Behandlungsareal fotodokumentiert. Die Parameter der einzelnen Behandlungen sind in $\bullet$ Tab. 1 aufgeführt. Wie bei der Behandlung von Tätowierungen mit gütegeschalteten Festkörperlasern üblich, wurde die kleinstmögliche Energie- dichte zur Behandlung eingestellt, die gerade noch das typische Geräusch der Fotodisruption in der Haut auslösen konnte. Da es nach der Fotodisruption zu Gasbläschenbildung im Gewebe kommt, kann das gleiche Areal nicht unmittelbar im Anschluss erneut behandelt werden. Die wiederholte Behandlung erfolgte in der Regel nach einer Wartezeit von 30 Minuten.

Für drei Patienten war dies die erste Laser-Behandlung ihrer Tätowierung, bei den drei restlichen Patienten waren 1, 2 bzw. 8 Einfachbehandlungen vorausgegangen, die 9 und mehr Wochen zurücklagen.

Zur Auswertung der fotografisch dokumentierten Aufhellung der so behandelten Tätowierungen wurde eine 101-stufige Grauwertskala verwendet, bei der Schwarz den Wert von 100 und Weiß (=komplette Aufhellung) den Wert von Null erhielt. Um die Zuordnung der Grauwerte (Restpigmente) der in der Haut befindlichen Tätowierungen zu erleichtern, wurden die Grauwertfelder jeweils zur Hälfte mit einem hautfarbenen Braunton unterlegt $($ Abb. 1). Die Auswertung der Patientenfotos mit Festlegung der entsprechenden Grauwerte wurde von 2 Personen unabhängig ermittelt und der jeweilige Mittelwert dokumentiert (๑ Tab.2). 
Tab.2 Grauwerte (bestimmt mittels Grauwerttabelle, siehe $\bullet$ Abb. 1) der Tätowierungspigmente vor und nach einer Vierfachbehandlung im Vergleich zu einer Einfachbehandlung mit einem gütegeschalteten Nd:YAG-Laser (Wellenlänge 1,064 nm).

\begin{tabular}{|c|c|c|c|c|c|c|}
\hline Pat.-Nr. & $\begin{array}{l}\text { Intervall zwischen } \\
\text { Behandlung und } \\
\text { Auswertung } \\
\text { (Wochen) }\end{array}$ & $\begin{array}{l}\text { Grauwert vor } \\
\text { der Behandlung }\end{array}$ & $\begin{array}{l}\text { Grauwert nach } \\
\text { einer Vierfach- } \\
\text { behandlung } \\
\text { (Probeareal) }\end{array}$ & $\begin{array}{l}\text { Aufhellung nach } \\
\text { einer Vierfach- } \\
\text { behandlung (\%) }\end{array}$ & $\begin{array}{l}\text { Grauwert nach } \\
\text { einer Einfach- } \\
\text { behandlung } \\
\text { (Kontrollareal) }\end{array}$ & $\begin{array}{l}\text { Aufhellung nach } \\
\text { einer Einfach- } \\
\text { behandlung (\%) }\end{array}$ \\
\hline 1 & 8 & 80 & 34 & 57,5 & 65 & 18,8 \\
\hline 2 & 16 & 81 & 13 & 84,0 & 40 & 50,6 \\
\hline 3 & 39 & 85 & 64 & 24,7 & 74 & 12,9 \\
\hline 4 & 14 & 78 & 62 & 20,5 & 72 & 7,7 \\
\hline 5 & 14 & 67 & 46 & 31,3 & 60 & 10,4 \\
\hline 6 & 9 & 82 & 69 & 15,9 & 71 & 13,4 \\
\hline Median & 13,7 & 80,5 & 54,0 & 28,0 & 68,0 & 13,2 \\
\hline SD & & 6,2 & 21,5 & 26,5 & 12,7 & 15,9 \\
\hline
\end{tabular}

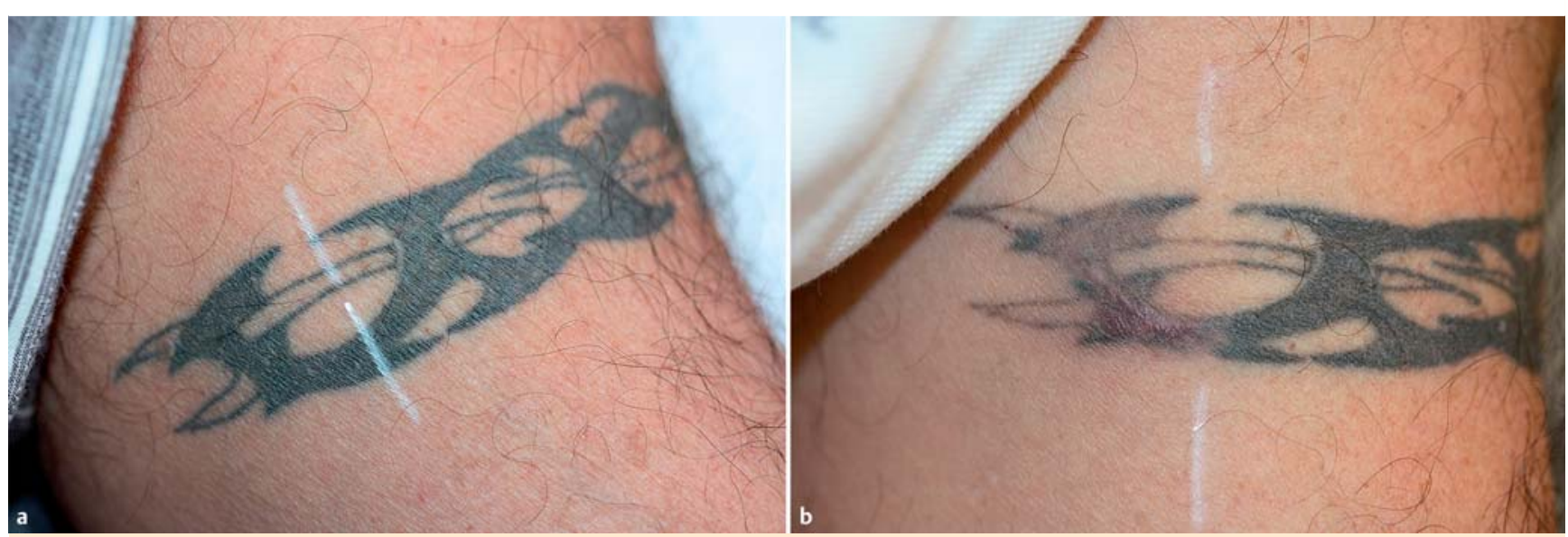

Abb.2 Professionelle Tätowierung vor (links) und 14 Wochen nach Vierfach- bzw. Einfachbehandlung mit gütegeschaltetem Nd:YAG-Laser (rechts); im Probeareal der Mehrfachbehandlung (Abschluss der Tätowierung links des Trennstrichs) zeigt sich eine deutlich bessere Aufhellung der Tätowierung (im Vergleich zur Einfachbehandlung), aber auch eine hypertrophe Narbe (Patient 4).

\section{Ergebnisse}

Die erzielte Aufhellung durch die einmalige Mehrfach- bzw. Einfachbehandlung der Tätowierungen wurde im Mittel nach 13,7 Wochen ausgewertet. Im Probeareal der Mehrfachbehandlung wurde eine Aufhellung von $28 \%$ erreicht, im Kontrollareal betrug die Aufhellung 13,2\%. Die Einzelwerte der 6 Patienten sind in - Tab.2 dargestellt. Ausgehend von einer Pigmentdichte der Tätowierungen von 80,5\% Schwarzanteil vor der Behandlung waren im Kontrollareal nach der Einfachbehandlung noch $68 \%$ Schwarzanteil verblieben, während nach einer Vierfachbehandlung die restlichen Schwarzanteile 54\% betrugen. Der Aufhellungseffekt einer Tätowierungsbehandlung mit gütegeschaltetem Nd:YAG-Laser konnte somit durch die Vierfachbehandlung im Mittel verdoppelt werden. Die bessere Aufhellung durch die Vierfachbehandlung konnte bei allen Patienten beobachtet werden, wenn auch in unterschiedlichem Ausmaß.

Hinsichtlich unerwünschter Wirkungen waren bei 4 der 6 Patienten in den Probearealen der Mehrfachbehandlung Nebenwirkungen (Erythem, Strukturveränderung, Narben) aufgetreten ( $\bullet$ Tab.3), während die nur einfach behandelten Areale nebenwirkungsfrei behandelt wurden ( $\bullet$ Abb. 2). Die höhere Belastung der Haut in den mehrfach behandelten Probearealen zeigte sich schon während der Mehrfachbehandlung mit stärkerem Erythem, Ödem, Purpura und Erosionen der Epidermis. Am Folgetag war es bei Patient $6 \mathrm{zu}$ einer deutlichen Blasenbildung gekommen (৫ Abb.3).

\section{Besprechung}

Eine Mehrfachbehandlung von Tätowierungen mit gütegeschaltetem Nd:YAG-Laser verbessert die Aufhellung, die durch eine Behandlungssitzung erreicht werden kann. Dies wurde erstmals durch Kossida bei 18 Tätowierungen von 12 Patienten durch Behandlung mit einem gütegeschalteten Alexandritlaser gezeigt [5] und später durch Reddy mit gütegeschaltetem Rubin- und Nd: YAG-Laser bestätigt [6], wobei in letzterer Arbeit zusätzlich gezeigt werden konnte, dass durch die topische Anwendung von Perfluorodecalin die Wartezeit zwischen den Mehrfachbehandlungen wegfallen kann.

Die vorliegende Kasuistik zeigt ebenfalls eine bessere Aufhellung der Tätowierungen durch die Verwendung der Mehrfachbehandlung, allerdings fällt die Verbesserung der Tätowierungsentfernung deutlich geringer aus, als bei den amerikanischen Kollegen: Kossida beschreibt eine vollständige Entfernung bei 61\% der Tätowierungen, Reddy gibt eine 51-75\%ige Aufhellung an, jeweils erzielt durch nur eine Behandlungssitzung, während wir bei den ersten 6 Patienten mit der Vierfachbehandlung eine mittlere Aufhellung von $28 \%$ erzielten (nur bei den Patienten 1 und 2 lag die Aufhellung über 50\%). Die Aufhellung der übrigen Tätowierung mit Einfachbehandlung beschreibt Kossida mit 18\% (bei professionellen Tätowierungen), bei uns lag die Aufhellung nach Einmalbehandlung bei $13,2 \%$. 


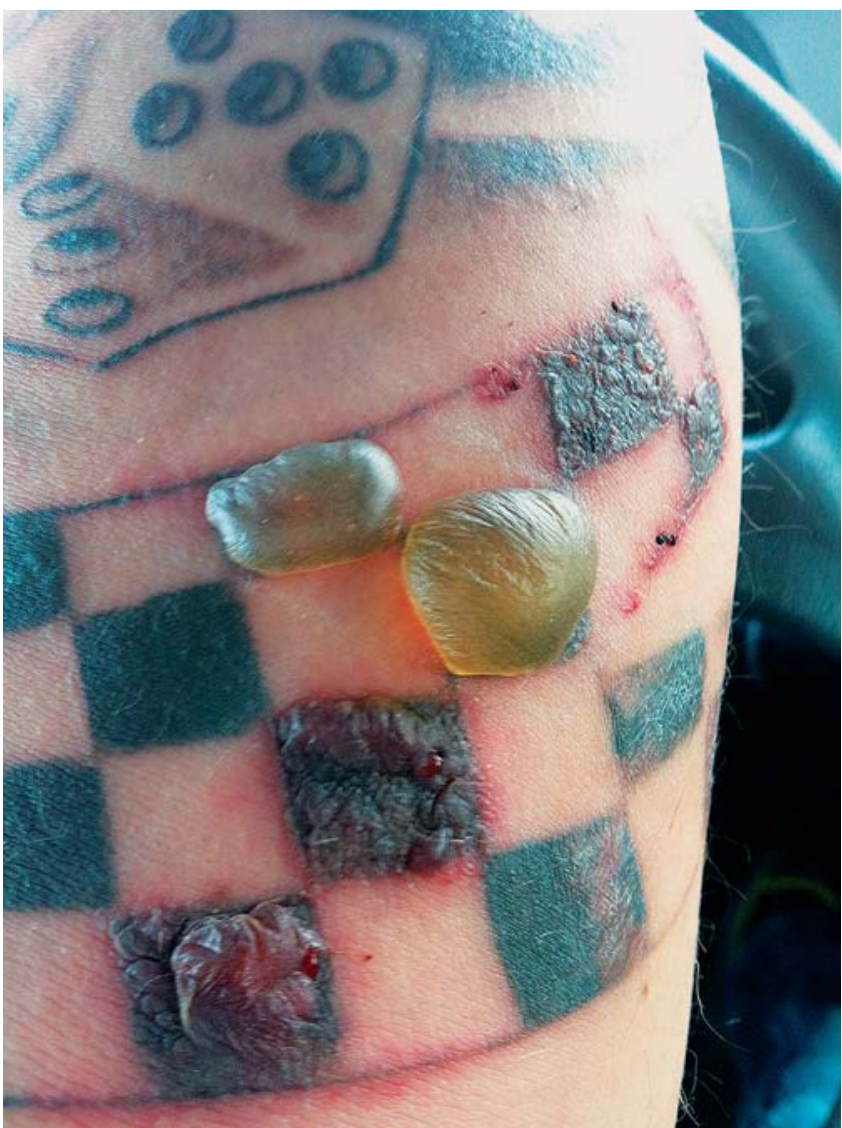

Abb.3 Ausgeprägte Blasenbildung einen Tag nach Mehrfachbehandlung einer Tätowierung mit gütegeschaltetem Nd:YAG-Laser (Patient 6).

Die Wirkung der mehrfachen Behandlungsdurchgänge auf die Zielstruktur ist noch spekulativ, wird aber durch histologische Untersuchungen in der Arbeit von Kossida gestützt: Durch geringere Gasbläschenbildung wird das Laserlicht der nachfolgenden Behandlungsdurchgänge weniger gestreut und gelangt so zu tiefer liegenden Pigmentpartikeln und kann diese dann ebenfalls zertrümmern. So werden bei jedem Durchgang tiefere Schichten erreicht.

Mehrere Behandlungsdurchgänge belasten jedoch auch stärker das übrige Gewebe. Beide Autoren berichten aber, dass keine unerwünschten Wirkungen aufgetreten seien, während bei unseren ersten 6 Fällen nur 2 Patienten im Probeareal keine Nebenwirkungen aufwiesen, die anderen 4 zeigten unerwünschte Wirkungen: Erytheme, Texturänderungen und Narben ( Tab.3). Aufgrund dieser hohen Nebenwirkungsfrequenz haben wir die Vierfachbehandlung von Tätowierungen abgebrochen.

Der Nachbeobachtungszeitraum zur Erfassung von Nebenwirkungen betrug bei Reddy nur 2 bis 4 Wochen nach der Behandlung, was für die Erfassung von Narbenbildungen zu kurz ist. Bei uns betrug das Intervall zur Nachuntersuchung 8 bis 39 Wochen (im Median 13,7 Wochen). Kossida gibt einen Nachuntersuchungszeitraum von 12 Wochen an.

Zur Frage einer möglichen Überdosierung ist anzumerken, dass der Medianwert der Energiedichte der einzelnen Behandlungssequenzen bei unseren Patienten mit 4 bis $5,8 \mathrm{~J} / \mathrm{cm}^{2}$ leicht über dem Bereich von 3-5J/ $\mathrm{cm}^{2}$ lag, den Reddy für die Behandlung angab. Zudem hatten unsere zwei nebenwirkungsfreien Fälle die höchsten Energiedichten während der Vierfachbehandlungen (Medianwerte der 4 Durchgänge: Patient $1=8,5 \mathrm{~J} / \mathrm{cm}^{2}$, Patient 5
Tab.3 Unerwünschte Wirkungen der Vierfachbehandlung von Tätowierungen im Vergleich zu einer Einfachbehandlung mit einem gütegeschalteten Nd:YAG-Laser (Wellenlänge 1,064 nm).

\begin{tabular}{|lll|}
\hline Pat.-Nr. & Unerwünschte Wirkungen der & \\
\cline { 2 - 3 } & Vierfachbehandlung & Einfachbehandlung \\
\hline 1 & keine & keine \\
\hline 2 & Erythem (gering, flächig) & keine \\
\hline 3 & Narbe? & keine \\
\hline 4 & Narbe (hypertroph) & keine \\
\hline 5 & keine & keine \\
\hline 6 & Erythem (mittel, flächig), & keine \\
\hline
\end{tabular}

$=7,3 \mathrm{~J} / \mathrm{cm}^{2}$ ), sodass eine Überdosierung als Grund für die aufgetretenen Nebenwirkungen eher unwahrscheinlich ist. Momentan sehen wir die hohe Frequenz der Mehrfachbehandlung (vierfache Behandlung) als mögliche Quelle für das hohe Nebenwirkungspotenzial, sodass wir diese neue Methode der Tätowierungsentfernung künftig allenfalls mit Dreifachbehandlungen fortführen werden. Ein zusätzliches Risiko könnte auch die Verwendung des tiefer penetrierenden Nd:YAG-Lasers bei größerem Strahldurchmesser sein (Kossida benutzte einen Alexandritlaser mit $3 \mathrm{~mm}$ Strahldurchmesser, Reddy einen Rubin- und auch einen Nd:YAGLaser, letzteren mit $4 \mathrm{~mm}$ Strahldurchmesser und Energiedichten zwischen $3-5 \mathrm{~J} / \mathrm{cm}^{2}$ ).

Insgesamt ist der Behandlungsaufwand bei Mehrfachbehandlungen deutlich höher, zum einen durch die mehrfach durchgeführte Behandlung, zum anderen aber auch durch die Wartezeit zwischen den Behandlungen, bis die Weißverfärbung (Gasbläschen) verschwunden ist. Die jeweils 20 Minuten Wartezeit zwischen den Mehrfachbehandlungen lässt sich offenbar vermeiden, wenn eine Ausleitung der Gasbläschen angeregt wird, wie der Einsatz von Perfluorodecalin zeigt [6]. Dadurch würde sich der zeitliche Mehraufwand einer Mehrfachbehandlung in Grenzen halten.

\section{Fazit für die Praxis}

Zur Verkürzung der Gesamtbehandlungszeit einer Tätowierungsentfernung scheint die Mehrfachbehandlung während einer Behandlungssitzung ein erfolgversprechender Weg. Solange jedoch nur wenige Studien zu dieser Behandlungsmodifikation vorliegen, sollte eine zu hohe Frequenz der Mehrfachbehandlung vermieden werden. Da bei unseren vierfachbehandelten Fällen das Narbenrisiko (unabhängig von der verwendeten Energiedichte) deutlich gegenüber einer Einfachbehandlung erhöht war, empfehlen wir derzeit allenfalls eine Dreifachbehandlung. Empfehlenswert ist auch, die Mehrfachbehandlung zunächst nur in einem Teil der Tätowierung vorzunehmen (Probeareal) und eine Nachbeobachtungszeit von mindestens 3 Monaten einzuhalten, bevor die gesamte Fläche der Mehrfachbehandlung unterzogen wird. Weitere Studien müssen herausfinden, wie viele Wiederholungsbehandlungen im Rahmen einer Mehrfachbehandlung und welcher Abstand zwischen den Behandlungssitzungen optimal ist, differenziert nach Art und Farbe der Tätowierungen, Hauttyp und Lasergerät. 


\section{Interessenkonflikt}

$\nabla$

Die Autoren geben an, dass keine Interessenkonflikte bestehen.

Abstract

\section{Multiple Exposure for Tattoo Removal - Better Clearance with Higher Risk of Scarring? \\ $\nabla$}

Introduction: Q-switched laser tattoo removal is safe and effective, but tedious because of many repeated treatments. To improve the clearance of tattoos, a technical modification was introduced with four repeated exposures during one treatment session. This report summarizes our own experiences with this multi-pass method.

Patients and method: Six patients with professional tattoos were treated with 4 repeated exposures in a test area, whereas the remainder of the tattoo was treated with normal single exposure by Q-switched Nd:YAG laser $\left(1.064 \mathrm{~nm}\right.$, Spectra ${ }^{\mathrm{TM}}$, Lutronic Cooperation). Three patients were treated for the first time. Using photo documentation the amount of black tattoo pigment was compared by measuring it with a step tablet (101 graded scale) before and at least 8 weeks after the treatment, by two independent investigators.

Results: All patients showed better clearance by repeated exposures compared to single exposure ( $28 \%$ vs. $13.2 \%$ ). The content of black tattoo pigment decreased from $80.5 \%$ at the beginning to $54 \%$ after the 4 treatment passes compared to $68 \%$ after a sin- gle treatment pass. On average the clearance was doubled in the test area. However, 4 out of 6 patients experienced side effects in the test area (erythema, textural changes and scars) whereas the control area had no side effects.

Conclusion: We can confirm the better clearance of tattoos by multiple treatment passes, although the effect was not as pronounced as described earlier. In view of the side effects we cannot recommend this new technique unless further studies are made exploring the optimal number of passes and the interval between the treatment sessions.

\section{Literatur}

1 Choudhary S, Elsaie ML, Leiva A et al. Lasers for tattoo removal: a review. Lasers Med Sci 2010; 25: 619-627

2 Ho DD, London R, Zimmerman GB et al. Laser-tattoo removal - a study of the mechanism and the optimal treatment strategy via computer simulations. Lasers Surg Med 2002; 30: 389-397

3 Bencini PL, Cazzaniga S, Tourlaki A et al. Removal of tattoos by q-switched laser - Variables influencing outcome and sequelae in a large cohort of treated patients. Arch Dermatol 2012; 148: $1364-$ 1369

4 Kirby W, Desai A, Desai $T$ et al. The Kirby-Desai scale: A proposed scale to assess tattoo-removal treatments. J Clin Aesthetic Dermatol 2009; 2: $32-37$

5 Kossida T, Rigopoulos D, Katsambas A et al. Optimal tattoo removal in a single laser session based on the method of repeated exposures. J Am Acad Dermatol 2012; 66: $271-277$

6 Reddy KK, Brauer JA, Anolik $R$ et al. Topical Perfluorodecalin resolves immediate whitening reactions and allows rapid effective multiple pass treatment of tattoos. Lasers Surg Med 2013; 45: 76-80 